\title{
On Casimir Operators of Conformal Galilei Algebras
}

\author{
Fahad Alshammari ${ }^{1,2}$, Phillip S. Isaac ${ }^{1}$ and Ian Marquette ${ }^{1}$ \\ ${ }^{1}$ School of Mathematics and Physics, The University of Queensland, St Lucia QLD \\ 4072, Australia \\ ${ }^{2}$ Department of Mathematics, Prince Sattam Bin Abdulaziz University, Saudi Arabia.
}

Email: fahad.alshammari@uqconnect.edu.au,psi@maths.uq.edu.au, i.marquette@uq.edu.au

\begin{abstract}
In previous work, we introduced an algorithm that utilises differential operator realisations to find polynomial Casimir operators of Lie algebras. In this article we build on this work by applying the algorithm to several classes of finite dimensional conformal Galilei algebras with central extension. In these cases we highlight the utility of an algebra anti-automorphism, and give relevant details through key examples.
\end{abstract}

\section{Introduction}

The role of Casimir operators of Lie algebras is undeniably important in certain areas of physics. For example, based on Lie symmetries of certain quantum Hamiltonians in fundamental cases, the energy spectrum can be expressed in terms of the eigenvalues of the Casimir elements on an irreducible submodule of the Hilbert space. Explicit expressions of Casimir operators of semisimple Lie algebras are well known. Such formulae for the Casimir operators of semisimple Lie algebras have been obtained by many authors with some key developments being given in [1-4]. However, for non-semisimple Lie algebras, they remain largely unexplored due to the complex nature of the algebraic structure itself.

Conformal Galilei groups and their Lie algebras are a class of nonrelativistic algebra [5]. Physical applications associated with these Galilei algebras include both classical and quantum mechanics [6], nonrelativistic spacetime and gravity [7-9], fluid dynamics [10], nonrelativistic holography and electrodynamics [11-14]. Conformal Galilei algebras form a class of non-semisimple Lie algebras, with each member characterised by two parameters $d$ (positive integer) and $\ell$ (positive integer or positive half-integer). The corresponding algebra is referred to as the conformal Galilei algebra with rational dynamical exponent $[5$, $8,15,16]$. The smallest instance of $\ell=\frac{1}{2}$ is known in the literature [17] as the Schrödinger algebra. The conformal Galilei algebra with higher value of $\ell$ is studied from physical and mathematical points of view $[5,8,11,17-26]$. In many cases it has been observed that physical systems having a connection with $\ell$-conformal Galilei $\left(\ell>\frac{1}{2}\right)$ algebra are described by Lagrangians or Hamiltonians with higher order derivatives. Also, there is a connection with this algebra as a symmetry algebra for generalised oscillator systems such as the Pais-Uhlenbeck oscillator [27].

The purpose of this paper is two fold: firstly, we use an efficient and functional algorithm, as presented in [28] and code using symbolic software to show how a structural map, namely an algebra anti-automorphism, along with a triangular decomposition of 
the Lie algebra, can be combined to simplify the algorithm. Secondly, we apply the algorithm to the conformal Galilei algebra for which, in some cases, explicit expressions for the Casimir operators have not been determined previously. We present certain explicit formulas for the case of dimension $d=1,2$ and arbitrary $\ell$.

The contents of this paper are organized as follows. In the next section we give a short review of generators of the $\ell$-conformal Galilei algebra and its central extensions. In section 3 and 4 we apply our direct approach using differential operator realisations to the conformal Galilei algebra with central extension for the case of $d=1$ and half-odd integer $\ell$, and the case of $d=2$ and arbitrary values of $\ell$. We highlight the advantage of the structural map in further reducing the amount of computation.

\section{The conformal Galilei algebra}

The class of Lie algebras known as the Conformal Galilei algebras have two types of central extension according to the values of $d$ and $\ell$. In the literature, it is not surprising that there are many results on central extensions of various classes of Lie algebras. Several authors have studied the existence of central extensions in the context of conformal Galilei algebras $[11,13,18,25,29-33]$.

Various results have already appeared in the literature concerning explicit expressions for Casimir operators for the cases $d=1,2,3$ and $\ell=\frac{1}{2}$ (e.g. see [20,26,34,35]). With the help of the orbit method, Casimir operators of conformal Galilei algebras with mass extension have been found in [21] for the case of $d=3$ and half-odd integer $\ell$. For other values of $d \geq 1$ and half-odd integer $\ell \geq \frac{3}{2}$, even in the case $d=1,2$, the Casimir operators have not been given in explicit detail. The current article has the modest goal of filling this gap for $d=1,2$, and various values of $\ell$ which allow a central extension. In doing so, we discover certain structural aspects of the conformal Galilei algebras which can improve the functionality of the algorithm presented in [28].

More general cases corresponding to $d \geq 2$ and half-odd integer and integer values $\ell \geq \frac{3}{2}$ will be the subject of future work.

Let us introduce the finite-dimensional $\ell$-conformal Galilei algebra without central extension in $d$-dimensional space, denoted $\mathfrak{g}_{\ell}(d)$, is a semi-direct sum of

$$
\mathfrak{s l}(2) \oplus \mathfrak{s o}(d) \boxplus \mathfrak{R}^{(2 \ell+1) d},
$$

where $\mathfrak{R}^{(2 \ell+1) d}$ is the abelian ideal relating to a space-translations, Galilean boosts and higher order accelerations. This algebra is parametrized by $d$ and $\ell$ which take on values

$$
d=1,2,3, \ldots, \quad \ell=\frac{1}{2}, 1, \frac{3}{2}, 2, \frac{5}{2}, \ldots
$$

Generally, the $\mathfrak{g}_{\ell}(d)$ is specified by the following generators( see $\left.[11,18-20]\right)$.

$$
P_{n, i}, H, D, C, J_{i j}=-J_{j i} ; n=0,1,2, \ldots, 2 \ell ; i, j=1,2, \ldots, d .
$$

The table below shows these generators can be classified as a set of transformations in the context of Galilean relativity (i.e. before the introduction of Einstein's special relativity) $[5,25]$. 
Table 1: Coordinate transformations in Galilean relativity

\begin{tabular}{|l|l|l|l|}
\hline Coordinate transformations & Linearisation & Generator & Physical description \\
\hline$\left(x_{i} \rightarrow x_{i}+\epsilon x_{j}, x_{j} \rightarrow x_{j}-\epsilon x_{i}\right)$ & $-x_{i} \frac{\partial}{\partial x_{j}}+x_{j} \frac{\partial}{\partial x_{i}}$ & $J_{i j}$ & rotations \\
\hline$(\mathrm{t} \rightarrow \mathrm{t}+\epsilon)$ & $\frac{\partial}{\partial t}$ & $H$ & time translation \\
\hline$\left(t \rightarrow(1-2 \epsilon) t, x_{i} \rightarrow(1-2 \ell \epsilon) x_{i}\right)$ & $-2 t \frac{\partial}{\partial t}-2 \ell x_{i} \frac{\partial}{\partial x_{i}}$ & $D$ & dilatation \\
\hline$\left(t \rightarrow(1+\epsilon t) t, x_{i} \rightarrow(1+2 \ell \epsilon t) x_{i}\right)$ & $t^{2} \frac{\partial}{\partial t}+2 \ell t x_{i} \frac{\partial}{\partial x_{i}}$ & $C$ & conformal transformation \\
\hline$\left(x_{i} \rightarrow x_{i}+\epsilon\right)$ & $\frac{\partial}{\partial x_{i}}$ & $P_{0, i}$ & space translation \\
$\left(x_{i} \rightarrow x_{i}-\epsilon t\right)$ & $-t \frac{\partial}{\partial x_{i}}$ & $P_{1, i}$ & Galilean boost \\
$\left(x_{i} \rightarrow x_{i}+\epsilon t^{2}\right)$ & $t^{2} \frac{\partial}{\partial x_{i}}$ & $P_{2, i}$ & acceleration \\
$\left(x_{i} \rightarrow x_{i}+\epsilon(-t)^{n}\right)$ & $(-t)^{n} \frac{\partial}{\partial x_{i}}$ & $P_{n, i}, n>2$ & higher derivative transfor- \\
& & & mation \\
\hline
\end{tabular}

The above generators satisfy the following non-trivial commutation relations

$$
\begin{aligned}
{[D, H] } & =2 H,[D, C]=-2 C,[C, H]=D, \\
{\left[H, P_{n, i}\right] } & =-n P_{n-1, i},\left[D, P_{n, i}\right]=2(\ell-n) P_{n, i},\left[C, P_{n, i}\right]=(2 \ell-n) P_{n+1, i}, \\
{\left[J_{i j}, P_{n, k}\right] } & =\delta_{i k} P_{n, j}-\delta_{j k} P_{n, i},\left[J_{i j}, J_{k \ell}\right]=\delta_{i k} J_{j \ell}+\delta_{j \ell} J_{i k}-\delta_{i \ell} J_{j k}-\delta_{j k} J_{i \ell} .
\end{aligned}
$$

The conformal Galilei algebra with central extension, denoted $\hat{\mathfrak{g}}_{\ell}(d)$, has two types of central extension [25]:

(1) A central extension exists for any dimension of spacetime and $\ell \in \mathbb{N}+\frac{1}{2}$

$$
\left[P_{m, i}, P_{n, j}\right]=\delta_{i j} \delta_{m+n, 2 \ell} I_{m} M, \quad I_{m}=(-1)^{m+\ell+1 / 2}(2 \ell-m) ! m !,
$$

where $M$ is central.

(2) The so-called exotic extension, which exists only for $\mathrm{d}=2$ and integer values of $\ell \in \mathbb{N}$

$$
\left[P_{m, i}, P_{n, j}\right]=\epsilon_{i j} \delta_{m+n, 2 \ell} I_{m} \Theta, \quad I_{m}=(-1)^{m}(2 \ell-m) ! m ! .
$$

Here $\Theta$ is central and $\epsilon_{i j}$ is the antisymmetric tensor with $\epsilon_{12}=1$. Notice that the dimension of the conformal Galilei algebra involving central extension $\hat{\mathfrak{g}}_{\ell}(d)$ is given by $2 d \ell+\frac{d(d+1)}{2}+4$.

\section{Conformal Galilei algebra with $d=1$ and $\ell \geq \frac{3}{2}$}

First recall the formula by Beltrametti and Blasi [36] for the number of generalised Casimir operators of a Lie algebra $\mathfrak{g}$,

$$
\operatorname{dim}(\mathfrak{g})-\operatorname{rank}(C(\mathfrak{g}))
$$

where $C(\mathfrak{g})$ denotes the commutator table of $\mathfrak{g}$ treated as a numerical matrix with generic entries. In the cases below, we find this number of polynomial Casimir operators.

For $d=1$, the Beltrametti-Blasi formula predicts that there are two functionally independent Casimir operators for $\hat{\mathfrak{g}}_{\ell}(1)$. One is clearly the central element $M$. The other Casimir operator will be constructed via the algorithm, the details of which can be found 
in [28]. We do not repeat the technical details here, but will refer to the steps (A), (B), $\ldots$, , (I) as presented in that article.

Following the algorithm, we need to first establish the artificial relative dimensions of the basis elements, a type of grading of the Lie algebra in a certain basis, as discussed in [28]. We introduce artificial relative dimensions $\alpha$ and $\beta$ and express the dimensions of all generators in a consistent way with the commutation relations (1),(2) and (4). This leads to the following:

$$
\begin{aligned}
{\left[P_{n}\right] } & =\alpha^{\frac{2 \ell-2 n}{2 \ell}} \beta^{\frac{n}{2 \ell}},[H]=\alpha^{\frac{2}{2 \ell}} / \beta^{\frac{1}{2 \ell}} \\
{[C] } & =\beta^{\frac{1}{2 \ell}} / \alpha^{\frac{2}{2 \ell}},[D]=1,[M]=\beta
\end{aligned}
$$

where $n=0,1,2, \ldots, 2 \ell$.

This step is useful in simplifying the process of taking linear combinations of terms in steps (B) and (C) of the search algorithm.

We make an observation for low dimensional cases that the lowest order Casimir operators has dimensions coinciding with $M^{2}$, i.e., $[K]=\beta^{2}$. For artificial relative dimensions $\alpha^{x} \beta^{y}$ for example, we simply denote these in terms of the indices only, i.e. $(x, y)$. In such notation, $[K]=(0,2)$.

For any half-odd integer $\ell$, we then make the assumption that $[K]=(0,2)$. This greatly reduces number of terms in the expression for the Casimir operator. We find that this assumption works in our method for the conformal Galilei algebra cases.

Next, the algorithm requires the construction of a differential operator realisation, as outlined in step (D). Those realisations already have been given in the literature (see [11] for more detail). The triangular decomposition of $\hat{\mathfrak{g}}_{\ell} \equiv \hat{\mathfrak{g}}_{\ell}(1)$ is given by

$$
\hat{\mathfrak{g}}_{\ell}=\hat{\mathfrak{g}}_{\ell}^{+} \oplus \hat{\mathfrak{g}}_{\ell}^{0} \oplus \hat{\mathfrak{g}}_{\ell}^{-},
$$

where,

$$
\hat{\mathfrak{g}}_{\ell}^{+}=\left\langle H, P_{0}, P_{1}, \ldots, P_{\ell-\frac{1}{2}}\right\rangle, \quad \hat{\mathfrak{g}}_{\ell}^{0}=\langle D, M\rangle, \quad \hat{\mathfrak{g}}_{\ell}^{-}=\left\langle C, P_{\ell+\frac{1}{2}}, P_{\ell+\frac{3}{2}}, \ldots, P_{2 \ell}\right\rangle,
$$

denoting $\langle\cdots\rangle$ for the span, and where $\delta$ is a free parameter related to the conformal weight of $D$. The realisations of $\hat{\mathfrak{g}}_{\ell}^{0}$ are given by

$$
D=\delta-2 t \frac{\partial}{\partial t}-\sum_{j=0}^{\ell-\frac{1}{2}} 2(\ell-j) x_{j} \frac{\partial}{\partial x_{j}}, \quad M=m,
$$

while the realisations of $\hat{\mathfrak{g}}_{\ell}^{+}$are represented as

$$
H=-\frac{\partial}{\partial t}, \quad P_{k}=-\sum_{j=0}^{k}\left(\begin{array}{c}
k \\
j
\end{array}\right) t^{k-j} \frac{\partial}{\partial x_{j}},
$$

where $k=0,1, \ldots, \ell-\frac{1}{2}$. The realisations of $\hat{\mathfrak{g}}_{\ell}^{-}$as

$$
\begin{aligned}
C & =t D+t^{2} \frac{\partial}{\partial t}+\frac{m}{2}\left[\left(\ell+\frac{1}{2}\right) !\right]^{2} x_{\ell-\frac{1}{2}}^{2}-\sum_{j=0}^{\ell-\frac{3}{2}}(2 \ell-j) x_{j} \frac{\partial}{\partial x_{j+1}} \\
P_{k} & =m \sum_{j=2 \ell-k}^{\ell-\frac{1}{2}}\left(\begin{array}{c}
k \\
2 \ell-j
\end{array}\right) I_{2 \ell-j} t^{k-2 \ell+j} x_{j}-\sum_{j=0}^{\ell-\frac{1}{2}}\left(\begin{array}{c}
k \\
j
\end{array}\right) t^{k-j} \frac{\partial}{\partial x_{j}}
\end{aligned}
$$


for $k=\ell+\frac{1}{2}, \ldots, 2 \ell$.

Note that the $\hat{\mathfrak{g}}_{\ell}(1)$ algebra is spanned by $2 \ell+5$ generators. For our purpose, it is convenient to fix the ordering of generators as

$$
\left\{M, P_{0}, P_{1}, \ldots, P_{\ell-\frac{3}{2}}, H, P_{\ell-\frac{1}{2}}, D, P_{\ell+\frac{1}{2}}, C, P_{\ell+\frac{3}{2}}, \ldots, P_{2 \ell}\right\}
$$

We proceed by applying the steps (E), (F) and (G) of the search algorithm to construct the Casimir operators. In fact, we have explicitly checked our results for $\hat{\mathfrak{g}}_{\ell}(1)$ and values

$\ell=\frac{3}{2}, \frac{5}{2}, \ldots, \frac{17}{2}$. Our algorithm has found Casimir operators with the same artificial relative dimension as the central element $M^{2}$, i.e. $(0,2)$. In addition, this algorithm shows that there is only one candidate Casimir operator in the case of arbitrary $\hat{\mathfrak{g}}_{\ell}(1)$ (in reference to step $(\mathrm{H})$ of the algorithm).

Before we present the output of the algorithm, we need to first introduce a useful lemma that makes use of an algebra anti-automorphism, which reduces a large amount of calculations.

\subsection{Utility of an algebra anti-automorphism for $d=1$}

The aim of this section is to prove an assertion concerning an algebra anti-automorphism for $d=1$. In the context of conformal Galilei algebras, the following lemma shows that we may only need to consider verifying the commutator of the proposed Casimir operator with a subset of basis vectors. Specifically for the case $d=1$, we define the involution anti-automorphism of the conformal Galilei algebra as [18]

$$
\omega(H)=C, \omega(C)=H, \omega(D)=D, \quad \omega(M)=M, \quad \omega\left(P_{n}\right)=P_{2 \ell-n} .
$$

\section{Lemma 1}

$$
\text { If } \omega(K)=K \text { and }[K, H]=0=\left[K, P_{2 \ell}\right] \text {, then }[K, X]=0, \forall X \in \hat{\mathfrak{g}}_{\ell}(1) \text {. }
$$

Proof: The proof is straightforward, using the commutation relations (1),(2),(4) and the Jacobi identity.

The main results are summarised below, corresponding to different cases.

\subsection{1 $\hat{\mathfrak{g}}_{\frac{3}{2}}(1)$}

As already discussed, for this case the Beltrametti-Blasi formula (6) implies that there exist two Casimir operators for $\hat{\mathfrak{g}}_{\frac{3}{2}}(1)$. One is clearly the central element $M$. By applying the algorithm, the search for a quartic Casimir operator with artificial relative dimensions $(0,2)$ was found

$$
\begin{aligned}
K & =-6 M^{2} D+M^{2} D^{2}-4 M^{2} H C-\frac{7}{2} M P_{0} P_{3}+\frac{5}{2} M P_{1} P_{2}-2 M\left(H P_{1} P_{3}+P_{0} P_{2} C\right) \\
& +2 M\left(H P_{2}^{2}+P_{1}^{2} C\right)+M P_{0} D P_{3}-M P_{1} D P_{2}+\frac{1}{4} P_{0}^{2} P_{3}^{2}-\frac{3}{2} P_{0} P_{1} P_{2} P_{3}-\frac{3}{4} P_{1}^{2} P_{2}^{2} \\
& +\left(P_{1}^{3} P_{3}+P_{0} P_{2}^{3}\right) .
\end{aligned}
$$


To check the expression $K$ in (15) is a Casimir operator, we employ the result of Lemma 1. It is clear that using the involution anti-automorphism in (13), it straightforward to confirm that $\omega(K)=K$. It only remains to check the following relations

$$
[K, H]=0=\left[K, P_{3}\right] .
$$

Without including all the details of the calculation, we have that

$$
\begin{aligned}
{[K, H] } & =(-12+4+8) M^{2} H+(4-4) M^{2} H D+\left(\frac{-21}{2}+\frac{5}{2}-2-2+12\right) M P_{0} P_{2} \\
& +(5-2-3) M P_{1}^{2}+(2-2) M P_{0} H P_{3}+(-6+8-2) M H P_{1} P_{2}+(3-3) P_{1}^{3} P_{2} \\
& +(3-2-1) M P_{0} D P_{2}+(2-2) M P_{1}^{2} D+(4-4) M P_{0} P_{1} C+\left(\frac{6}{4}-\frac{3}{2}\right) P_{0}^{2} P_{2} P_{3} \\
& +\left(-\frac{9}{2}-\frac{3}{2}+6\right) P_{0} P_{1} P_{2}^{2}+(3-3) P_{0} P_{1}^{2} P_{3} \\
& =0 .
\end{aligned}
$$

Also,

$$
\begin{aligned}
{\left[K, P_{3}\right] } & =(18-9-21+12) M^{2} P_{3}+(-6+6) M^{2} D P_{3}+(12-12) M^{2} P_{2} C \\
& +(6+3-9) M P_{1} P_{2} P_{3}+(-6+6) M P_{2}^{3}+(-3+3) M P_{0} P_{3}^{2} \\
& =0
\end{aligned}
$$

It is easily verified that the conditions of Lemma 1 are satisfied, and hence that $K$ in equation (15) is indeed a Casimir operator of $\hat{\mathfrak{g}}_{\frac{3}{2}}(1)$.

It is worth remarking that the Casimir operator $K$ in (15) has two types of terms. One type is self-conjugate under $\omega$ (e.g. $\left.\omega\left(M P_{0} P_{3}\right)=M P_{0} P_{3}\right)$, and the other type involves a conjugate pair under $\omega$ (e.g. $\left.\omega\left(M H P_{1} P_{3}\right)=M P_{0} P_{2} C\right)$.

\subsection{2 $\hat{\mathfrak{g}}_{\frac{5}{2}}(1)$}

Repeating the same process with the case $\ell=\frac{5}{2}$, there are still two functionally independent Casimir operators. One is clearly the central element $M$. By applying the algorithm, a quartic Casimir operator was found with artificial relative dimensions $(0,2)$ as follows:

$$
\begin{aligned}
K & =132 M^{2} D-12 M^{2} D^{2}+48 M^{2} H C-M P_{0} P_{5}+28 M P_{1} P_{4}-10 M P_{2} P_{3}-2 M\left(P_{1} H P_{5}\right. \\
& \left.+P_{0} C P_{4}\right)+8 M\left(H P_{2} P_{4}+P_{1} P_{3} C\right)-6 M\left(H P_{3}^{2}+P_{2}^{2} C\right)+M P_{0} D P_{5}-3 M P_{1} D P_{4} \\
& +2 M P_{2} D P_{3}-\frac{1}{48} P_{0}^{2} P_{5}^{2}+\frac{5}{24} P_{0} P_{1} P_{4} P_{5}-\frac{1}{12} P_{0} P_{2} P_{3} P_{5}-\frac{3}{16} P_{1}^{2} P_{4}^{2}+\frac{19}{12} P_{1} P_{2} P_{3} P_{4} \\
& +\frac{2}{3} P_{2}^{2} P_{3}^{2}-\frac{1}{3}\left(P_{1}^{2} P_{3} P_{5}+P_{0} P_{2} P_{4}^{2}\right)+\frac{1}{4}\left(P_{1} P_{2}^{2} P_{5}+P_{0} P_{3}^{2} P_{4}\right)-\left(P_{2}^{3} P_{4}+P_{1} P_{3}^{3}\right) .
\end{aligned}
$$

Similar to the case of $\hat{\mathfrak{g}}_{\frac{3}{2}}(1)$ in the previous subsection 3.1.1, we employ the result of Lemma 1. By using the involution anti-automorphism in (13), it is obvious that $\omega(K)=$ $K$. Now, in order to see the remaining conditions of the Lemma 1 are satisfied, we need 
to compute commutators of the Casimir operator $K$ in (16) with the generators $H$ and $P_{5}$. A straightforward computation shows that

$$
[K, H]=0=\left[K, P_{5}\right]
$$

All the conditions for the Lemma 1 are satisfied. Therefore, the expression $K$ in $(16)$ is indeed a Casimir operator of $\hat{\mathfrak{g}}_{\frac{5}{2}}(1)$.

\subsection{3 $\hat{\mathfrak{g}}_{\ell}(1), \ell \geq \frac{5}{2}$}

Using similar methods to subsections 3.1.1 and 3.1.2 we have explicitly checked the Casimir operators for half-odd integer values of $\ell$ up to $\frac{17}{2}$. It is clear from our computation that the case of $\ell=\frac{3}{2}$ is a special case. Extending to arbitrary half-odd integer value sof $\ell$, we summarise the results in the following theorem, the proof of which follows similar calculations to those already presented in subsections 3.1.1 and 3.1.2.

Theorem 1 For $\ell \geq \frac{5}{2}, \hat{\mathfrak{g}}_{\ell}(1)$ has a quartic Casimir operator of the form

$$
\begin{aligned}
K= & \alpha M^{2} D+\beta M^{2} D^{2}+\gamma M^{2} H C+\psi_{\ell-\frac{3}{2}} M\left(H P_{\ell-\frac{1}{2}} P_{\ell+\frac{3}{2}}+P_{\ell-\frac{3}{2}} P_{\ell+\frac{1}{2}} C\right) \\
& +\psi_{\ell-\frac{1}{2}} M\left(H P_{\ell+\frac{1}{2}}^{2}+P_{\ell-\frac{1}{2}}^{2} C\right)+\sum_{i=0}^{\ell-\frac{1}{2}} \varphi_{i} M A_{i}+\sum_{i=0}^{\ell-\frac{5}{2}} \psi_{i} M\left(B_{i}+C_{i}\right) \\
& +\sum_{i=0}^{\ell-\frac{1}{2}} \theta_{i} M D_{i}+\sum_{i=0}^{\ell-\frac{1}{2}} \sum_{j=i}^{\ell-\frac{1}{2}} \tau_{i j} X_{i j}+\sum_{i=0}^{\ell-\frac{3}{2}} \sum_{j=i}^{\ell-\frac{3}{2}} \lambda_{i j} Y_{i j},
\end{aligned}
$$

where,

$$
\begin{aligned}
& A_{i}=P_{i} P_{2 \ell-i}, B_{i}=P_{i+1} H P_{2 \ell-i}, C_{i}=P_{i} C P_{2 \ell-i-1}, D_{i}=P_{i} D P_{2 \ell-i}, \\
& X_{i j}=P_{i} P_{j} P_{2 \ell-j} P_{2 \ell-i}, \quad Y_{i j}=P_{i+1} P_{j+1} P_{2 \ell-j-2} P_{2 \ell-i}+P_{i} P_{j+2} P_{2 \ell-j-1} P_{2 \ell-i-1},
\end{aligned}
$$

and $\alpha, \beta, \gamma, \psi_{\ell-\frac{3}{2}}, \psi_{\ell-\frac{1}{2}}, \varphi_{i}, \psi_{i}, \theta_{i}, \tau_{i j}$, and $\lambda_{i j}$ are constants given by

$$
\begin{gathered}
\alpha=\left[\frac{1}{2}(-1)^{\ell+\frac{3}{2}}\left(\ell+\frac{1}{2}\right)^{2}(2 \ell-1) !-(-1)^{\ell+\frac{1}{2}}(2 \ell-1) !\right], \quad \beta=\frac{1}{2}(-1)^{\ell+\frac{1}{2}}(2 \ell-1) !, \\
\gamma=-2(-1)^{\ell+\frac{1}{2}}(2 \ell-1) !, \quad \psi_{\ell-\frac{1}{2}}=\frac{-(-1)^{\ell+\frac{3}{2}}(2 \ell-1) !}{\left[\left(\ell-\frac{1}{2}\right) !\right]^{2}}, \\
\psi_{i}=\frac{2(-1)^{2 \ell-i+2}(2 \ell-1) !}{(i) !(2 \ell-i-1) !}, \quad i=0, \ldots, \ell-\frac{3}{2}, \quad \varphi_{\ell-\frac{3}{2}}=\frac{-7(-1)^{\ell+\frac{1}{2}}\left(\ell-\frac{1}{2}\right)(2 \ell-1) !}{2\left(\ell-\frac{3}{2}\right) !\left(\ell+\frac{1}{2}\right) !}, \\
\varphi_{\ell-\frac{1}{2}}=\frac{(-1)^{\ell+\frac{1}{2}}\left(\ell+\frac{1}{2}\right)[5+4 \ell(\ell+1)](2 \ell-1) !}{8\left[\left(\ell+\frac{1}{2}\right) !\right]^{2}},
\end{gathered}
$$




$$
\begin{aligned}
& \varphi_{i}=\frac{(-1)^{i}\left[(-1)^{2 \ell+1} i(2 \ell+1)^{2}+\ell(7-4 \ell(\ell-1))\right](2 \ell-1) !}{4(i) !(2 \ell-i) !}, \quad i=0, \ldots, \ell-\frac{5}{2}, \\
& \theta_{i}=\frac{(-1)^{2 \ell-i+1}(2 \ell-2 i)(2 \ell-1) !}{(i) !(2 \ell-i) !}, \quad i=0, \ldots, \ell-\frac{1}{2} \\
& \tau_{\ell-\frac{1}{2} \ell-\frac{1}{2}}=\frac{-(-1)^{\ell+\frac{1}{2}}\left(\ell+\frac{3}{2}\right)(2 \ell-1) !}{2\left(\ell+\frac{1}{2}\right)\left(\ell-\frac{3}{2}\right) !\left(\ell+\frac{1}{2}\right) !\left[\left(\ell+\frac{1}{2}\right) !\right]^{2}}, \\
& \tau_{i i+1}=\frac{4(-1)^{\frac{1}{2}-\ell}\left[\frac{1}{2}(-1)^{2(\ell+i)} i(i-1-2 \ell)+(i-\ell)^{2}\right](2 \ell-1) !}{(i+1)(2 \ell-i) !(2 \ell-i-1) !(i !)^{2}}, \quad i=0, \ldots, \ell-\frac{3}{2}, \\
& \tau_{i i}=\frac{-2(-1)^{\frac{1}{2}-\ell}(i-\ell)^{2}(2 \ell-1) !}{(i !)^{2}[(2 \ell-i) !]^{2}}, \quad i=0, \ldots, \ell-\frac{3}{2} \text {, } \\
& \tau_{i j}=\frac{4(-1)^{\ell+i-j+\frac{5}{2}}\left[(-1)^{2 j} i-\ell\right](j-\ell)(2 \ell-1) !}{i !(j) !(2 \ell-i) !(2 \ell-j) !}, \quad i=0, \ldots, \ell-\frac{5}{2}, \quad j=i+2, \ldots, \ell-\frac{1}{2}, \\
& \lambda_{i \ell-\frac{3}{2}}=\frac{(-1)^{2 \ell+i-1}(2 \ell-1) !}{i !(2 \ell-i-1) !\left[\left(\ell-\frac{1}{2}\right) !\right]^{2}}, \quad i=0, \ldots, \ell-\frac{3}{2}, \\
& \lambda_{i j}=\frac{2(-1)^{\ell+i+j+\frac{1}{2}}(2 \ell-1) !}{i !(j+1) !(2 \ell-i-1) !(2 \ell-j-2) !}, \quad i=0, \ldots, \ell-\frac{5}{2}, j=i, \ldots, \ell-\frac{5}{2} .
\end{aligned}
$$

\section{Conformal Galilei algebras with $d=2$ and $\ell \geq 1$}

Here we show that the algorithm also applies to the construction of Casimir operators for the conformal Galilei algebra with the exotic central extension for $d=2$. Let us denote this algebra by $\hat{\mathfrak{g}}_{\ell}(2)$, where $\ell \in \mathbb{Z}_{+}$. Previously, these Casimir operators of $\hat{\mathfrak{g}}_{\ell}(2)$ have appeared in [21] and [33], and our results are consistent with these works.

A vector field realisation of the Conformal Galilei algebras with central extension has been found for $d=2$ and arbitrary values of $\ell$ [11]. This algebra has triangular decomposition which can be written as a direct sum of the vector space $\hat{\mathfrak{g}}_{\ell}=\hat{\mathfrak{g}}_{\ell}^{+} \oplus \hat{\mathfrak{g}}_{\ell}^{0} \oplus \hat{\mathfrak{g}}_{\ell}^{-}$, where

$$
\begin{array}{ll}
\hat{\mathfrak{g}}_{\ell}^{+}=\left\langle H, Q_{\ell}, Q_{n}, P_{n}\right\rangle, & n=0,1, \ldots, \ell-1, \\
\hat{\mathfrak{g}}_{\ell}^{0}=\langle D, J, \Theta\rangle, & \\
\hat{\mathfrak{g}}_{\ell}^{-}=\left\langle C, P_{\ell}, Q_{n}, P_{n}\right\rangle, & n=\ell+1, \ell+2, \ldots, 2 \ell .
\end{array}
$$

The following non-trivial commutation relations of the conformal Galilei algebra $\hat{\mathfrak{g}}_{\ell}(2)$ 
are given by

$$
\begin{aligned}
{[D, H] } & =2 H, \quad[D, C]=-2 C, \quad[C, H]=D \\
{\left[H, Q_{n}\right] } & =-n Q_{n-1}, \quad\left[H, P_{n}\right]=-n P_{n-1}, \\
{\left[D, Q_{n}\right] } & =2(\ell-n) Q_{n}, \quad\left[D, P_{n}\right]=2(\ell-n) P_{n}, \\
{\left[C, Q_{n}\right] } & =(2 \ell-n) Q_{n+1}, \quad\left[C, P_{n}\right]=(2 \ell-n) P_{n+1}, \\
{\left[J, Q_{n}\right] } & =Q_{n}, \quad\left[J, P_{n}\right]=-P_{n}, \quad\left[Q_{m}, P_{n}\right]=\delta_{m+n, 2 \ell} I_{m} \Theta .
\end{aligned}
$$

Note that $I_{m}$ is given in $(5)$.

Now, we work through the algorithm to produce the lowest order Casimir operators. First of all, the algorithm requires the use of differential operator realisations, described in step (D) of [28]. We use the realisations given in [11].

Explicitly, the realisations of $\hat{\mathfrak{g}}_{\ell}^{+}$are given by

$$
H=-\frac{\partial}{\partial t}, \quad Q_{n}=-\sum_{k=0}^{n}\left(\begin{array}{c}
n \\
k
\end{array}\right) t^{k} \frac{\partial}{\partial x_{n-k}}, \quad P_{n}=-\sum_{k=0}^{n}\left(\begin{array}{c}
n \\
k
\end{array}\right) t^{k} \frac{\partial}{\partial y_{n-k}},
$$

for $n=0,1, \ldots, \ell-1$. For $\hat{\mathfrak{g}}_{\ell}^{0}$, we have

$$
\begin{aligned}
D & =\delta-2 t \frac{\partial}{\partial t}-\sum_{n=0}^{\ell-1} 2(\ell-n)\left(x_{n} \frac{\partial}{\partial x_{n}}+y_{n} \frac{\partial}{\partial y_{n}}\right) \\
J & =r-\sum_{n=0}^{\ell} x_{n} \frac{\partial}{\partial x_{n}}+\sum_{n=0}^{\ell-1} y_{n} \frac{\partial}{\partial y_{n}}, \quad \Theta=-\theta
\end{aligned}
$$

where $\delta$ is a free parameter, and the realisations of $\hat{\mathfrak{g}}_{\ell}^{-}$that we use are given by

$$
\begin{gathered}
C=t D+t^{2} \frac{\partial}{\partial t}-\ell I_{\ell+1} \theta x_{\ell} y_{\ell-1}-\sum_{n=0}^{\ell-1}(2 \ell-n) x_{n} \frac{\partial}{\partial x_{n+1}}-\sum_{n=0}^{\ell-2}(2 \ell-n) y_{n} \frac{\partial}{\partial y_{n+1}}, \\
Q_{n}=-\theta \sum_{k=0}^{n-\ell-1}\left(\begin{array}{c}
n \\
k
\end{array}\right) I_{n-k} t^{k} y_{2 \ell-n+k}-\sum_{k=n-\ell}^{n}\left(\begin{array}{c}
n \\
k
\end{array}\right) t^{k} \frac{\partial}{\partial x_{n-k}}, \\
P_{n}=\theta \sum_{k=0}^{n-\ell}\left(\begin{array}{c}
n \\
k
\end{array}\right) I_{n-k} t^{k} x_{2 \ell-n+k}-\sum_{k=n-\ell+1}^{n}\left(\begin{array}{c}
n \\
k
\end{array}\right) t^{k} \frac{\partial}{\partial y_{n-k}} .
\end{gathered}
$$

where $n=\ell+1, \ell+2, \ldots, 2 \ell$. Note that $\hat{\mathfrak{g}}_{\ell}(2)$ is spanned by $4 \ell+7$ elements. For our purpose, it is convenient to fix the ordering of those generators within elements of the enveloping algebra as

$$
\left\{\Theta, Q_{0}, P_{0}, Q_{1}, P_{1}, \ldots, Q_{\ell-1}, P_{\ell-1}, H, D, J, Q_{\ell}, P_{\ell}, C, Q_{\ell+1}, P_{\ell+1}, \ldots, Q_{2 \ell}, P_{2 \ell}\right\} .
$$

From the Lie bracket given in (18-22), we are able to introduce artificial relative dimensions $\alpha, \beta$ and $\gamma$ and express the dimensions of all generators in a consistent way with the commutation relations. This leads to the following:

$$
\begin{aligned}
{\left[P_{n}\right] } & =\frac{\alpha}{\gamma^{n}}, \quad\left[Q_{n}\right]=\frac{\beta}{\alpha} \gamma^{2 \ell-n},[C]=\frac{1}{\gamma}, \\
{[H] } & =\gamma,[D]=[J]=1,[\Theta]=\beta,
\end{aligned}
$$


where $n=0,1,2, \ldots, 2 \ell$. As before, we adopt the notation using only indices, so that if an element $A$ has artificial relative dimension $[A]=\alpha^{x} \beta^{y} \gamma^{z}$, we simply write the triple $[A]=(x, y, z)$.

For the conformal Galilei algebra $\hat{\mathfrak{g}}_{\ell}(2)$, we make the assumption (actually based on calculations for specific cases) that the lowest order Casimir operator has artificial relative dimensions the same as the central element $\Theta$, i.e. $(0,1,0)$ and $\Theta^{2}$ (artificial relative dimensions $(0,2,0))$. This greatly reduces number of terms in the expression for the Casimir operator. We find that this assumption works in our method for the conformal Galilei algebra cases.

We apply the steps $(\mathrm{E}),(\mathrm{F})$ and $(\mathrm{G})$ of the search algorithm by using Mathematica software in order to solve the corresponding linear algebraic equations. In the case $d=2$, the algorithm leads us to the candidate Casimir operators which arise from step $(\mathrm{H})$.

\subsection{Utility of an algebra anti-automorphism for $d=2$}

Before summarising the output of the algorithm, let us present a functional lemma, analogous to Lemma 1. It turns out for the case $d=2$, only three elements are needed to verify the relations of the Casimir operator. Define the map

$$
\begin{aligned}
& \omega(H)=C, \omega(C)=H, \quad \omega(D)=D, \quad \omega(J)=J \\
& \omega(\Theta)=\Theta, \quad \omega\left(P_{n}\right)=Q_{2 \ell-n}, \quad \omega\left(Q_{n}\right)=P_{2 \ell-n} .
\end{aligned}
$$

We have the following result, the proof of which is straightforward computation involving commutators and the Jacobi identity.

\section{Lemma 2}

If $\omega(K)=K$ and $[K, H]=[K, J]=\left[K, P_{2 \ell}\right]=\left[K, Q_{2 \ell}\right]=0$, then $[K, X]=0, \forall X \in \hat{\mathfrak{g}}_{\ell}(2)$.

It is easily seen that the Beltrametti-Blasi formula (6) implies that there exist three Casimir operators for $\hat{\mathfrak{g}}_{\ell}(2)$. One is obviously the exotic central extension $\Theta$. To obtain the other two Casimir operators, we proceed by applying the search algorithm to $\hat{\mathfrak{g}}_{\ell}(2)$.

In what follows, the let $K_{(r, s, t)}^{(n), \ell}$ denote a polynomial Casimir operator for $\hat{\mathfrak{g}}_{\ell}(2)$ of degree $n$ and relative dimension $(r, s, t)$.

\subsubsection{The $\hat{\mathfrak{g}}_{1}(2)$ case}

Using the algorithm to search for a quadratic Casimir operator of artificial relative dimensions $(0,1,0)$ yields the following:

$$
K_{(0,1,0)}^{(2), 1}=-\Theta J-\frac{1}{2} Q_{0} P_{2}-\frac{1}{2} P_{0} Q_{2}+Q_{1} P_{1}
$$


Next, we seek a quartic Casimir operator of artificial relative dimensions $(0,2,0)$. The algorithm indicates two candidates which are not actual Casimir operators, given by

$$
\begin{aligned}
K_{a} & =-3 \Theta^{2} D-6 \Theta^{2} J+\frac{1}{2} \Theta^{2} D^{2}-2 \Theta^{2} H C+2 \Theta^{2} J^{2}-4 \Theta Q_{0} P_{2}+2 \Theta H P_{1} Q_{2} \\
& -2 \Theta H Q_{1} P_{2}-\Theta P_{0} D Q_{2}+2 \Theta P_{0} Q_{1} C+\Theta Q_{0} D P_{2}-2 \Theta Q_{0} P_{1} C+2 P_{0} Q_{1}^{2} P_{2} \\
& +2 Q_{0} P_{1}^{2} Q_{2}-2 Q_{1}^{2} P_{1}^{2}-2 Q_{0} P_{0} Q_{2} P_{2}, \\
K_{b} & =-6 \Theta^{2} J+2 \Theta^{2} J^{2}-2 \Theta P_{0} Q_{2}-2 Q_{1}^{2} P_{1}^{2}-2 Q_{0} P_{0} Q_{2} P_{2}-\frac{1}{2} Q_{0}^{2} P_{2}^{2}-\frac{1}{2} P_{0}^{2} Q_{2}^{2} \\
& +2 Q_{0} Q_{1} P_{1} P_{2}+2 P_{0} Q_{1} P_{1} Q_{2} .
\end{aligned}
$$

Using the involution anti-automorphism given in (25), we see that $\omega\left(K_{a}\right)=K_{a}$ and $\omega\left(K_{b}\right)=K_{b}$. Following step (I), we take a linear combination of the above candidates

$$
K_{(0,2,0)}^{(4), 1}=\alpha K_{a}+\beta K_{b},
$$

and impose the relations $\left[K_{(0,2,0)}^{(4), 1}, H\right]=\left[K_{(0,2,0)}^{(4), 1}, J\right]=\left[K_{(0,2,0)}^{(4), 1}, P_{2}\right]=\left[K_{(0,2,0)}^{(4), 1}, Q_{2}\right]=0$. We find that

$$
K_{(0,2,0)}^{(4), 1}=K_{a}-K_{b}
$$

That is,

$$
\begin{aligned}
K_{(0,2,0)}^{(4), 1}= & -3 \Theta^{2} D+\frac{1}{2} \Theta^{2} D^{2}-2 \Theta^{2} H C+2 \Theta\left(H P_{1} Q_{2}+P_{0} Q_{1} C\right)-2 \Theta\left(H Q_{1} P_{2}+Q_{0} P_{1} C\right) \\
& -2\left(Q_{0} Q_{1} P_{1} P_{2}+P_{0} Q_{1} P_{1} Q_{2}\right)+2 \Theta P_{0} Q_{2}-4 \Theta Q_{0} P_{2}-\Theta P_{0} D Q_{2}+\Theta Q_{0} D P_{2} \\
& +2\left(P_{0} Q_{1}^{2} P_{2}+Q_{0} P_{1}^{2} Q_{2}\right)-Q_{0} P_{0} Q_{2} P_{2}+\frac{1}{2}\left(P_{0}^{2} Q_{2}^{2}+Q_{0}^{2} P_{2}^{2}\right) .
\end{aligned}
$$

Hence, by Lemma 2, we have the Casimir operator for $\hat{\mathfrak{g}}_{1}(2)$.

\subsubsection{The $\hat{\mathfrak{g}}_{2}(2)$ case}

In this case we expect three Casimir operators, one of which is $\Theta$. Using the algorithm, we find quadratic and quartic Casimir operators. The quadratic Casimir operator of artificial relative dimensions $(0,1,0)$ can be written as

$$
K_{(0,1,0)}^{(2), 2}=-\Theta J-\frac{1}{24} Q_{0} P_{4}-\frac{1}{24} P_{0} Q_{4}+\frac{1}{6} Q_{1} P_{3}+\frac{1}{6} P_{1} Q_{3}-\frac{1}{4} Q_{2} P_{2} .
$$


For the quartic Casimir operator of artificial relative dimensions $(0,2,0)$, the algorithm indicates two candidates as follows:

$$
\begin{aligned}
K_{a}= & -54 \Theta^{2} D+3 \Theta^{2} D^{2}+12 \Theta^{2} D J-12 \Theta^{2} H C+5 \Theta P_{0} Q_{4}-16 \Theta P_{1} Q_{3}-\Theta Q_{0} P_{4} \\
& +20 \Theta Q_{1} P_{3}+3 \Theta D Q_{2} P_{2}-6 \Theta\left(H P_{2} Q_{3}+P_{1} Q_{2} C\right)+6 \Theta\left(H Q_{2} P_{3}+Q_{1} P_{2} C\right) \\
& +2 \Theta\left(P_{1} H Q_{4}+P_{0} C Q_{3}\right)-2 \Theta\left(Q_{1} H P_{4}+Q_{0} C P_{3}\right)-\frac{1}{2} \Theta P_{0} D Q_{4}+\frac{3}{2} \Theta Q_{0} D P_{4} \\
& -4 \Theta Q_{1} D P_{3}+3\left(P_{1} Q_{2}^{2} P_{3}+Q_{1} P_{2}^{2} Q_{3}\right)+\left(P_{0} P_{2} Q_{3}^{2}+P_{1}^{2} Q_{2} Q_{4}+Q_{0} Q_{2} P_{3}^{2}+Q_{1}^{2} P_{2} P_{4}\right) \\
& +\frac{1}{3}\left(P_{0} Q_{1} Q_{3} P_{4}+Q_{0} P_{1} P_{3} Q_{4}\right)-\left(P_{0} Q_{2} Q_{3} P_{3}+Q_{1} P_{1} P_{2} Q_{4}\right)+\frac{1}{12}\left(P_{0}^{2} Q_{4}^{2}+Q_{0}^{2} P_{4}^{2}\right) \\
& -\left(Q_{0} P_{2} Q_{3} P_{3}+Q_{1} P_{1} Q_{2} P_{4}\right)+\frac{1}{3}\left(P_{1}^{2} Q_{3}^{2}+Q_{1}^{2} P_{3}^{2}\right)-\frac{2}{3} Q_{1} P_{1} Q_{3} P_{3}-\frac{1}{6} Q_{0} P_{0} Q_{4} P_{4} \\
& -\frac{2}{3}\left(P_{0} P_{1} Q_{3} Q_{4}+Q_{0} Q_{1} P_{3} P_{4}\right)+\frac{1}{3}\left(P_{0} Q_{1} P_{3} Q_{4}+Q_{0} P_{1} Q_{3} P_{4}\right) \\
& -3\left(P_{1} Q_{2} P_{2} Q_{3}+Q_{1} Q_{2} P_{2} P_{3}\right), \\
K_{b}= & -12 \Theta^{2} D+12 \Theta^{2} D J+2 \Theta P_{0} Q_{4}-4 \Theta P_{1} Q_{3}+2 \Theta Q_{0} P_{4}-4 \Theta Q_{1} P_{3}+3 \Theta D Q_{2} P_{2} \\
& +\frac{1}{2} \Theta P_{0} D Q_{4}-2 \Theta P_{1} D Q_{3}+\frac{1}{2} \Theta Q_{0} D P_{4}-2 \Theta Q_{1} D P_{3}
\end{aligned}
$$

It turns out neither of these are Casimir operators, but behave as such when restricted to the realisation. As in the $\hat{\mathfrak{g}}_{1}(2)$ case in the previous section, by imposing the conditions of Lemma in 2, we find

$$
K_{(0,2,0)}^{(4), 2}=K_{a}-K_{b}
$$

Specifically,

$$
\begin{aligned}
K_{(0,2,0)}^{(4), 2}= & -42 \Theta^{2} D+3 \Theta^{2} D^{2}-12 \Theta^{2} H C+2 \Theta\left(P_{1} H Q_{4}+P_{0} C Q_{3}\right)-2 \Theta\left(Q_{1} H P_{4}+Q_{0} C P_{3}\right) \\
& -6 \Theta\left(H P_{2} Q_{3}+P_{1} Q_{2} C\right)+6 \Theta\left(H Q_{2} P_{3}+Q_{1} P_{2} C\right)-3\left(Q_{1} Q_{2} P_{2} P_{3}+P_{1} Q_{2} P_{2} Q_{3}\right) \\
& +3 \Theta P_{0} Q_{4}-3 \Theta Q_{0} P_{4}-12 \Theta P_{1} Q_{3}+24 \Theta Q_{1} P_{3}-\Theta P_{0} D Q_{4}+\Theta Q_{0} D P_{4} \\
& +2 \Theta P_{1} D Q_{3}-2 \Theta Q_{1} D P_{3}+\frac{1}{3}\left(P_{0} Q_{1} Q_{3} P_{4}+Q_{0} P_{1} P_{3} Q_{4}\right)+3\left(P_{1} Q_{2}^{2} P_{3}+Q_{1} P_{2}^{2} Q_{3}\right) \\
& -\frac{1}{6} Q_{0} P_{0} Q_{4} P_{4}-\frac{2}{3} Q_{1} P_{1} Q_{3} P_{3}-\left(P_{0} Q_{2} Q_{3} P_{3}+Q_{1} P_{1} P_{2} Q_{4}\right)+\frac{1}{12}\left(P_{0}^{2} Q_{4}^{2}+Q_{0}^{2} P_{4}^{2}\right) \\
& -\frac{2}{3}\left(P_{0} P_{1} Q_{3} Q_{4}+Q_{0} Q_{1} P_{3} P_{4}\right)+\frac{1}{3}\left(P_{1}^{2} Q_{3}^{2}+Q_{1}^{2} P_{3}^{2}\right)-\left(Q_{0} P_{2} Q_{3} P_{3}+Q_{1} P_{1} Q_{2} P_{4}\right) \\
& +\frac{1}{3}\left(P_{0} Q_{1} P_{3} Q_{4}+Q_{0} P_{1} Q_{3} P_{4}\right)+\left(P_{0} P_{2} Q_{3}^{2}+P_{1}^{2} Q_{2} Q_{4}+Q_{0} Q_{2} P_{3}^{2}+Q_{1}^{2} P_{2} P_{4}\right) .
\end{aligned}
$$

It is easily verified that the conditions of Lemma in 2 are satisfied, and hence that $K_{(0,2,0)}^{(4), 2}$ is a Casimir operator for $\hat{\mathfrak{g}}_{2}(2)$. 


\subsubsection{The $\hat{\mathfrak{g}}_{3}(2)$ case}

At the risk of being monotonous, we look at one final value $\ell=3$ before summarising in the general case. For the case $\ell=3$, we expect three functionally independent Casimir operators, as in the previous cases $\ell=1$ and $\ell=2$. In applying our algorithm, a quadratic Casimir operator was found with artificial relative dimensions $(0,1,0)$ as follows:

$$
\begin{aligned}
K_{(0,1,0)}^{(2), 3} & =-\Theta J-\frac{1}{720} Q_{0} P_{6}-\frac{1}{720} P_{0} Q_{6}+\frac{1}{120} Q_{1} P_{5}+\frac{1}{120} P_{1} Q_{5}-\frac{1}{48} Q_{2} P_{4}-\frac{1}{48} P_{2} Q_{4} \\
& +\frac{1}{36} Q_{3} P_{3} .
\end{aligned}
$$

A functionally independent quartic Casimir operator was also found with artificial relative dimensions $(0,2,0)$ :

$$
\begin{aligned}
& K_{(0,2,0)}^{(4), 3}=-1560 \Theta^{2} D+60 \Theta^{2} D^{2}-240 \Theta^{2} H C+8 \Theta P_{0} Q_{6}-24 \Theta P_{1} Q_{5}+60 \Theta P_{2} Q_{4}-8 \Theta Q_{0} P_{6} \\
& +24 \Theta Q_{1} P_{5}-120 \Theta Q_{2} P_{4}+2 \Theta\left(P_{1} H Q_{6}+P_{0} C Q_{5}\right)-10 \Theta\left(P_{2} H Q_{5}+P_{1} C Q_{4}\right) \\
& +20 \Theta\left(H P_{3} Q_{4}+P_{2} Q_{3} C\right)-2 \Theta\left(Q_{1} H P_{6}+Q_{0} C P_{5}\right)+10 \Theta\left(Q_{2} H P_{5}+Q_{1} C P_{4}\right) \\
& -20 \Theta\left(H Q_{3} P_{4}+Q_{2} P_{3} C\right)-\Theta P_{0} D Q_{6}+4 \Theta P_{1} D Q_{5}-5 \Theta P_{2} D Q_{4}+\Theta Q_{0} D P_{6} \\
& -4 \Theta Q_{1} D P_{5}+5 \Theta Q_{2} D P_{4}-\frac{5}{3}\left(Q_{2} Q_{3} P_{3} P_{4}+P_{2} Q_{3} P_{3} Q_{4}\right)+\frac{1}{60}\left(P_{0} Q_{1} Q_{5} P_{6}\right. \\
& \left.+Q_{0} P_{1} P_{5} Q_{6}\right)+\frac{5}{12}\left(P_{1} Q_{2} Q_{4} P_{5}+Q_{1} P_{2} P_{4} Q_{5}\right)+\frac{5}{3}\left(P_{2} Q_{3} Q_{3} P_{4}+Q_{2} P_{3} P_{3} Q_{4}\right) \\
& -\frac{1}{120} Q_{0} P_{0} Q_{6} P_{6}-\frac{2}{15} Q_{1} P_{1} Q_{5} P_{5}-\frac{5}{24} Q_{2} P_{2} Q_{4} P_{4}-\frac{1}{12}\left(P_{0} Q_{2} Q_{5} P_{5}+Q_{1} P_{1} P_{4} Q_{5}\right) \\
& -\frac{5}{6}\left(P_{1} Q_{3} Q_{4} P_{4}+Q_{2} P_{2} P_{3} Q_{5}\right)+\frac{1}{240}\left(P_{0}^{2} Q_{6}^{2}+Q_{0}^{2} P_{6}^{2}\right)-\frac{1}{20}\left(P_{0} P_{1} Q_{5} Q_{6}+Q_{0} Q_{1} P_{5} P_{6}\right) \\
& +\frac{1}{24}\left(P_{0} P_{2} Q_{4} Q_{6}+Q_{0} Q_{2} P_{4} P_{6}\right)+\frac{1}{15}\left(P_{1}^{2} Q_{5}^{2}+Q_{1}^{2} P_{5}^{2}\right)-\frac{7}{12}\left(P_{1} P_{2} Q_{4} Q_{5}+Q_{1} Q_{2} P_{4} P_{5}\right) \\
& +\frac{5}{48}\left(P_{2}^{2} Q_{4}^{2}+Q_{2}^{2} P_{4}^{2}\right)-\frac{1}{12}\left(Q_{0} P_{2} Q_{5} P_{5}+Q_{1} P_{1} Q_{4} P_{6}\right)+\frac{1}{6}\left(Q_{0} P_{3} Q_{4} P_{5}+Q_{1} P_{2} Q_{3} P_{6}\right) \\
& -\frac{5}{6}\left(Q_{1} P_{3} Q_{4} P_{4}+Q_{2} P_{2} Q_{3} P_{5}\right)+\frac{1}{30}\left(P_{0} Q_{1} P_{5} Q_{6}+Q_{0} P_{1} Q_{5} P_{6}\right)-\frac{1}{24}\left(P_{0} Q_{2} P_{4} Q_{6}\right. \\
& \left.+Q_{0} P_{2} Q_{4} P_{6}\right)+\frac{1}{6}\left(P_{1} Q_{2} P_{4} Q_{5}+Q_{1} P_{2} Q_{4} P_{5}\right)+\frac{1}{12}\left(P_{0} P_{2} Q_{5}^{2}+P_{1}^{2} Q_{4} Q_{6}+Q_{0} Q_{2} P_{5}^{2}\right. \\
& \left.+Q_{1}^{2} P_{4} P_{6}\right)-\frac{1}{6}\left(P_{0} P_{3} Q_{4} Q_{5}+P_{1} P_{2} Q_{3} Q_{6}+Q_{0} Q_{3} P_{4} P_{5}+Q_{1} Q_{2} P_{3} P_{6}\right)+\frac{5}{6}\left(P_{1} P_{3} Q_{4}^{2}\right. \\
& \left.+P_{2}^{2} Q_{3} Q_{5}+Q_{1} Q_{3} P_{4}^{2}+Q_{2}^{2} P_{3} P_{5}\right)+\frac{1}{6}\left(P_{0} Q_{3} P_{4} Q_{5}+P_{1} Q_{2} P_{3} Q_{6}\right) .
\end{aligned}
$$

The form of $K_{(0,2,0)}^{(4), 3}$ given above is slightly different to the quartic Casimir operators given for the cases $\ell=1$ and $\ell=2$. It turns out, however, that this form prevails for other values $\ell \geq 3$ as we see in the next section.

\subsubsection{The $\hat{\mathfrak{g}}_{\ell}(2)$ case}

Here we summarise the explicit expressions of the Casimir operators determined by the algorithm for the exotic conformal Galilei algebra in $d=2$ and arbitrary integer $\ell$. In 
fact, this algebra has three functionally independent Casimir operators. Clearly, the central extension $\Theta$ is a Casimir operator. The others are quadratic and quartic Casimir operators. The following expression of quadratic Casimir operator of artificial relative dimension $(0,1,0)$ was deduced from the forms of the Casimir operators given in (26), (28) and (31), but the proof is a straightforward but tedious exercise in commutation relations.

Theorem 2 For $d=2$ and $\ell \geq 1$, the quadratic Casimir operator of the exotic conformal Galilei algebra has the form

$$
K_{(0,1,0)}^{(2), \ell}=-\Theta J+\sum_{m=0}^{\ell-1} \frac{(-1)^{m+1}}{m !(2 \ell-m) !}\left(Q_{m} P_{2 \ell-m}+P_{m} Q_{2 \ell-m}\right)+(-1)^{\ell+1} \frac{1}{(\ell !)^{2}} Q_{\ell} P_{\ell} .
$$

For the quartic Casimir operator of the exotic conformal Galilei algebra $\hat{\mathfrak{g}}_{\ell}(2)$, where $\ell \geq 3$. It is clearly from our results thta the form of the quartic Casimir operators for $\ell=1$ and $\ell=2$ are special cases of $\hat{\mathfrak{g}}_{\ell}(2)$. The following expression was deduced from the forms of the Casimir operators corresponding to specific values of $\ell$, which are shown to be genuine Casimir operators by the tedious task of verifying commutation relations.

Theorem 3 For $d=2$ and arbitrary integer $\ell \geq 3$, there is a quartic Casimir operator of artificial relative dimension $(0,2,0)$ given by

$$
\begin{aligned}
& K_{(0,2,0)}^{(4), \ell}=\alpha \Theta^{2} D+\beta \Theta^{2} D^{2}+\gamma \Theta^{2} H C+\psi_{\ell-1} \Theta\left(H P_{\ell} Q_{\ell+1}+P_{\ell-1} Q_{\ell} C\right) \\
& +\psi_{\ell-1}^{\prime} \Theta\left(H Q_{\ell} P_{\ell+1}+Q_{\ell-1} P_{\ell} C\right)+\delta_{\ell-1}\left(Q_{\ell-1} Q_{\ell} P_{\ell} P_{\ell+1}+P_{\ell-1} Q_{\ell} P_{\ell} Q_{\ell+1}\right) \\
& +\sum_{i=0}^{\ell-1} \varphi_{i} \Theta A_{i}+\sum_{i=0}^{\ell-1} \varphi_{i}^{\prime} \Theta A_{i}^{\prime}+\sum_{i=0}^{\ell-2} \psi_{i} \Theta\left(B_{i}+C_{i}\right)+\sum_{i=0}^{\ell-2} \psi_{i}^{\prime} \Theta\left(B_{i}^{\prime}+C_{i}^{\prime}\right) \\
& +\sum_{i=0}^{\ell-1} \phi_{i} \Theta D_{i}+\sum_{i=0}^{\ell-1} \phi_{i}^{\prime} \Theta D_{i}^{\prime}+\sum_{i=0}^{\ell-1} \rho_{i} S_{i}+\sum_{i=0}^{\ell-1} \eta_{i} R_{i}+\sum_{i=0}^{\ell-2} \mu_{i} T_{i}+\sum_{i=0}^{\ell-1} \sum_{j=i}^{\ell-1} \tau_{i j} X_{i j} \\
& +\sum_{i=0}^{\ell-2} \sum_{j=i}^{\ell-2} \lambda_{i j} Y_{i j}+\sum_{i=0}^{\ell-2} \sum_{j=i}^{\ell-2} \zeta_{i j} Z_{i j}+\sum_{i=0}^{\ell-2} \sum_{j=i}^{\ell-2} \omega_{i j} U_{i j}+\sum_{i=0}^{\ell-3} \sum_{j=i}^{\ell-3} \varepsilon_{i j} V_{i j},
\end{aligned}
$$

where

$$
\begin{gathered}
A_{i}=P_{i} Q_{2 \ell-i}, A_{i}^{\prime}=Q_{i} P_{2 \ell-i}, B_{i}=P_{i+1} H Q_{2 \ell-i}, B_{i}^{\prime}=Q_{i+1} H P_{2 \ell-i}, \\
C_{i}=P_{i} C Q_{2 \ell-i-1}, C_{i}^{\prime}=Q_{i} C P_{2 \ell-i-1}, D_{i}=P_{i} D Q_{2 \ell-i}, D_{i}^{\prime}=Q_{i} D P_{2 \ell-i}, \\
S_{i}=P_{i} Q_{i+1} Q_{2 \ell-i-1} P_{2 \ell-i}+Q_{i} P_{i+1} P_{2 \ell-i-1} Q_{2 \ell-i}, R_{i}=Q_{i} P_{i} Q_{2 \ell-i} P_{2 \ell-i}, \\
T_{i}=P_{i} Q_{i+2} Q_{2 \ell-i-1} P_{2 \ell-i-1}+Q_{i+1} P_{i+1} P_{2 \ell-i-2} Q_{2 \ell-i}, \\
X_{i j}=P_{i} P_{j} Q_{2 \ell-j} Q_{2 \ell-i}+Q_{i} Q_{j} P_{2 \ell-j} P_{2 \ell-i}, \\
Y_{i j}=Q_{i} P_{j+2} Q_{2 \ell-j-1} P_{2 \ell-i-1}+Q_{i+1} P_{j+1} Q_{2 \ell-j-2} P_{2 \ell-i}, \\
Z_{i j}=P_{i} Q_{j+1} P_{2 \ell-j-1} Q_{2 \ell-i}+Q_{i} P_{j+1} Q_{2 \ell-j-1} P_{2 \ell-i},
\end{gathered}
$$




$$
\begin{gathered}
U_{i j}=P_{i} P_{j+2} Q_{2 \ell-j-1} Q_{2 \ell-i-1} P_{i+1} P_{j+1} Q_{2 \ell-j-2} Q_{2 \ell-i}+Q_{i} Q_{j+2} P_{2 \ell-j-1} P_{2 \ell-i-1}+Q_{i+1} Q_{j+1} P_{2 \ell-j-2} P_{2 \ell-i} \\
V_{i j}=P_{i} Q_{j+3} P_{2 \ell-j-2} Q_{2 \ell-i-1}+P_{i+1} Q_{j+2} P_{2 \ell-j-3} Q_{2 \ell-i}
\end{gathered}
$$

and $\alpha, \beta, \gamma, \psi_{\ell-1}, \psi_{\ell-1}^{\prime}, \delta_{\ell-1}, \varphi_{i}, \varphi_{i}^{\prime}, \psi_{i}, \psi_{i}^{\prime}, \phi_{i}, \phi_{i}^{\prime}, \rho_{i}, \eta_{i}, \mu_{i}, \tau_{i j}, \lambda_{i j}, \zeta_{i j}, \omega_{i j}, \varepsilon_{i j}$ are constants. The form of these are given by

$$
\begin{aligned}
& \alpha=\left[(-1)^{2 \ell+1} \ell(\ell+1)(2 \ell-1) !-(2 \ell-1) !\right], \quad \beta=\frac{1}{2}(2 \ell-1) !, \\
& \gamma=-2(2 \ell-1) !, \quad \delta_{\ell-1}=\frac{-2(2 \ell-1) !}{[(\ell) !(\ell-1) !]^{2}}, \\
& \varphi_{i}=\frac{-(-1)^{i}\left[1+(-1)^{2 \ell}(i-\ell)\right](\ell+1)(2 \ell) !}{(i) !(2 \ell-i) !}, \quad i=0, \ldots, \ell-2 \text {, } \\
& \varphi_{i}^{\prime}=\frac{(-1)^{i}\left[-1+(-1)^{2 \ell}(\ell-i)\right](\ell+1)(2 \ell) !}{(i) !(2 \ell-i) !}, \quad i=0, \ldots, \ell-2, \\
& \varphi_{\ell-1}=\frac{-2(-1)^{\ell}(2 \ell-1) !}{[(\ell-1) !]^{2}}, \quad \varphi_{\ell-1}^{\prime}=\frac{4(-1)^{\ell}(2 \ell-1) !}{[(\ell-1) !]^{2}}, \\
& \psi_{i}=\frac{2(-1)^{2 \ell-i}(2 \ell-1) !}{(i) !(2 \ell-i-1) !}, \quad i=0, \ldots, \ell-1, \\
& \psi_{i}^{\prime}=\frac{-2(-1)^{2 \ell-i}(2 \ell-1) !}{(i) !(2 \ell-i-1) !}, \quad i=0, \ldots, \ell-1, \\
& \phi_{i}=\frac{-(-1)^{2 \ell-i}(2 \ell-2 i)(2 \ell-1) !}{(i) !(2 \ell-i) !}, \quad i=0, \ldots, \ell-1, \\
& \phi_{i}^{\prime}=\frac{(-1)^{2 \ell-i}(2 \ell-2 i)(2 \ell-1) !}{(i) !(2 \ell-i) !}, \quad i=0, \ldots, \ell-1 \\
& \rho_{i}=\frac{-2(-1)^{2 \ell-2 i-1}(2 \ell-1) !}{[(i) !(2 \ell-i-1) !]^{2}}, \quad i=0, \ldots, \ell-1 \\
& \mu_{i}=\frac{2(-1)^{2 \ell-2 i-1}(2 \ell-1) !}{(i) !(i+1) !(2 \ell-i-1) !(2 \ell-i-2) !}, \quad i=0, \ldots, \ell-2, \\
& \eta_{i}=\frac{-4(-1)^{2 \ell-2 i}\left[(-1)^{2 i}(1+i-\ell)-1\right](i-\ell)(2 \ell-1) !}{[(i) !(2 \ell-i-1) !]^{2}}, \quad i=0, \ldots, \ell-1 \\
& \tau_{\ell-1 \ell-1}=\frac{-(2 \ell-1) ![(\ell+2)(\ell-1) !(\ell) !-\ell(\ell-2) !(\ell+1) !]}{(\ell-2) !(\ell) ![(\ell-1) !(\ell+1) !]^{2}}, \quad \tau_{00}=\frac{1}{2(2 \ell-1) !},
\end{aligned}
$$




$$
\begin{gathered}
\tau_{i i+1}=\frac{2(-1)^{2 \ell-2 i}\left[i+i^{2}-2 i \ell+2 \ell^{2}\right](i+1)(i-2 \ell)(2 \ell-1) !}{[(i+1) !(2 \ell-i) !]^{2}}, \quad i=0, \ldots, \ell-3, \\
\tau_{i+1 i+1}=\frac{2(-1)^{2 \ell-2 i}(i-\ell+1)^{2}(2 \ell-1) !}{[(i+1) !(2 \ell-i-1) !]^{2}}, \quad i=0, \ldots, \ell-3 \\
\tau_{i j+2}=\frac{4(-1)^{2 \ell-i-j-1}(\ell-i)(j+2-\ell)(2 \ell-1) !}{(i) !(j+2) !(2 \ell-i) !(2 \ell-j-2) !}, \quad i=0, \ldots, \ell-3, \quad j=i, \ldots, \ell-3, \\
\lambda_{i j}=-\omega_{i j}=\frac{2(-1)^{2 \ell-i-j-1}(2 \ell-1) !}{(i) !(j+1) !(2 \ell-i-1) !(2 \ell-j-2) !}, \quad i=0, \ldots, \ell-2, j=i, \ldots, \ell-2, \\
\varsigma_{i j}=\frac{-4(-1)^{2 \ell+i-j-1}(\ell-i)(\ell-j-1)(2 \ell-1) !}{(i) !(j+1) !(2 \ell-i) !(2 \ell-j-1) !}, i=0, \ldots, \ell-2, j=i, \ldots, \ell-2, \\
\varepsilon_{i j}=\frac{2(-1)^{\ell-i-j-2}(2 \ell-1) !}{(i) !(j+2) !(2 \ell-i-1) !(2 \ell-j-3) !}, \quad i=0, \ldots, \ell-3, j=i, \ldots, \ell-3,
\end{gathered}
$$

\section{Conclusion}

In this short paper, we have constructed, via the search algorithm presented in [28], the Casimir operators of certain conformal Galilei algebras with central extensions. The focus was on the cases corresponding to underlying spatial dimensions $d=1$ and $d=2$, and different values of $\ell$ (positive half-odd integer or integer). The modest goal of the article was to draw attention to the fact that the algorithm can be augmented by making use of a structural feature of these algebras, namely the algebra anti-automorphism $\omega$, that allowed us to restrict the search algorithm even further to only look for Casimir operators conjugate under the action of $\omega$.

It would be interesting to look at further cases corresponding to higher spatial dimension $d \geq 2$ for arbitrary values of $\ell$. More generally, kinematical Lie algebras, among them the conformal and Schrodinger algebras, are still object of an ongoing classification (see in particular the recent works of Figueroa-O'Farrill and Andrzejewski [37-39]). The method developed in the current paper may enable further insight into the structure of Casimir operators of a wider class of non-semisimple Lie algebras.

\section{Acknowledgements:}

The research of FA is supported by Prince Sattam Bin Abdulaziz University. IM is supported by the Australian Research Council Discovery Grant DP160101376. PSI is supported by the Australian Research Council through Discovery Project DP150101294.

\section{References}

[1] Gruber B, and O’Raifeartaigh L, J. Math. Phys. 5 (1964) 1796.

[2] Okubo S, J. Math. Phys. 18 (1977) 2382. 
[3] Perelomov A M, and Popov V S, Math. USSR Izv. 2 (1968) 1313.

[4] Racah G, Phys. Rev. 76 (1949) 1365.

[5] Negro J, del Olmo M A and Rodriguez-Marco A, J. Math. Phys. 38 (1997) 3786.

[6] Alvarez P D, Gomis J, Kamimura K and Plyushchay M S, Ann. Phys. 322 (2007) 1556.

[7] Bagchi A, Phys. Rev. Lett. 105 (2010) 171601.

[8] Duval C and Horvathy P A, J. Phys. A: Math. Theor. 42 (2009) 465206.

[9] Hotta K, Kubota T and Nishinaka T, Nucl. Phys. B 838 (2010) 358.

[10] Zhang P and Horváthy P A, Eur. Phys. J. C 65 (2010) 607.

[11] Aizawa N, Kimura Y and Segar J, J. Phys. A: Math. Theor. 46 (2013) 405204.

[12] Le Bellac M and Lévy-Leblond J M, Nuv. Cim. B 14 (1973) 217.

[13] Lévy-Leblond, J M, Group theory and its applications(1971) Academic Press, New York.

[14] Negro J, Del Olmo M A and Rodriguez-Marco, A, J. Math. Phys. 38 (1997) 3810.

[15] Duval C and Horvathy P A, J. Phys. A: Math. Theor. 44 (2011) 335.

[16] Stichel P C and Zakrzewski W J, Entropy 15 (2013) 559.

[17] Niederer U, Helv. Phys. Acta 45 (1972) 802.

[18] Aizawa N, Isaac P S and Kimura Y, Int. J. Math. 23 (2012) 1250118.

[19] Aizawa N and Isaac P S, J. Phys. A: Math. Theor. 44 (2011) 035401.

[20] Aizawa N and Dobrev V K, Nucl. Phys. B 828 (2010) 581.

[21] Andrzejewski K, Gonera J and Maslanka P, Phys. Rev. D 86 (2012) 065009.

[22] Bacry H and Levy-Leblond J M, J. Math. Phys. 9 (1968) 1605.

[23] Campoamor-Stursberg R and Low S G, J. Phys. A: Math. Theor. 42 (2009) 065205.

[24] Dobrev V K, Doebner H D and Mrugalla C, Rep. Math. Phys. 39 (1997) 201.

[25] Martelli D and Tachikawa Y, JHEP 5 (2010) 1.

[26] Perroud M, Helv. Phys. Acta 50 (1977) 233.

[27] Andrzejewski K, Galajinsky A, Gonera J and Masterov I, Nucl. Phys. B 885 (2014) 150 . 
[28] Alshammari F, Isaac P S and Marquette I, J. Phys. A: Math. Theor. 51 (2018) 065206 .

[29] Bargmann V, Ann. Math. 59 (1954) 1.

[30] Hosseiny A, J. Math. Phys. 55 (2014) 061704.

[31] Lukierski J, Stichel P C and Zakrzewski W J, Ann. Phys. 260 (1997) 224.

[32] Lukierski J, Stichel P C and Zakrzewski W J, Phys. Lett. A 357 (2006) 1.

[33] Lukierski J, Stichel P C and Zakrzewski W J, Phys. Lett. B 650 (2007) 203.

[34] Campoamor-Stursberg R, J. Phys. A: Math. Gen. 38 (2005) 4187.

[35] Henkel M, Phys. Rev. Lett. 78 (1997) 1940.

[36] Beltrametti E G and Blasi A, Phys. Lett. 20 (1966) 62.

[37] Figueroa-O'Farrill J M, J. Math. Phys. 59 (2018) 061701.

[38] Andrzejewski T and Figueroa-O'Farrill J M, J. Math. Phys. 59 (2018) 061703.

[39] Figueroa-O'Farrill J M, arXiv:1809.03603 [hep-th] (2018). 